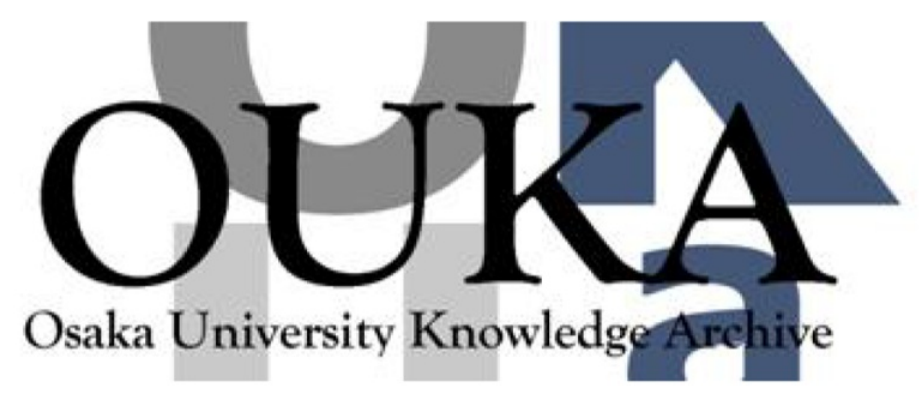

\begin{tabular}{|c|l|}
\hline Title & $\begin{array}{l}\text { Amplified spontaneous emission and lasing in } \\
\text { conducting polymers and fluorescent dyes in } \\
\text { opals as photonic crystals }\end{array}$ \\
\hline Author(s) & $\begin{array}{l}\text { Yoshino, K.; Tatsuhara, S. ; Kawagishi, Y. et } \\
\text { al. }\end{array}$ \\
\hline Citation & Applied Physics Letters. 74(18) p. 2590-p. 2592 \\
\hline Issue Date & $1999-03-03$ \\
\hline oaire:version & VoR \\
\hline URL & https://hdl. handle.net/11094/75840 \\
\hline rights & \\
\hline Note & \\
\hline
\end{tabular}

Osaka University Knowledge Archive : OUKA

https://ir. Library. osaka-u. ac. jp/

Osaka University 


\section{Amplified spontaneous emission and lasing in conducting polymers and fluorescent dyes in opals as photonic crystals}

Cite as: Appl. Phys. Lett. 74, 2590 (1999); https://doi.org/10.1063/1.123907

Submitted: 20 November 1998 . Accepted: 08 March 1999. Published Online: 27 April 1999

K. Yoshino, S. Tatsuhara, Y. Kawagishi, M. Ozaki, A. A. Zakhidov, and Z. V. Vardeny

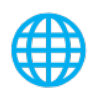

\section{ARTICLES YOU MAY BE INTERESTED IN}

Observation of inhibited spontaneous emission and stimulated emission of rhodamine 6C in polymer replica of synthetic opal

Applied Physics Letters 73, 3506 (1998); https://doi.org/10.1063/1.122819

Plasmonically enhanced diffusive and subdiffusive metal nanoparticle-dye random laser Applied Physics Letters 86, 131105 (2005); https://doi.org/10.1063/1.1894590

A comprehensive review of $\mathrm{ZnO}$ materials and devices

Journal of Applied Physics 98, 041301 (2005); https://doi.org/10.1063/1.1992666

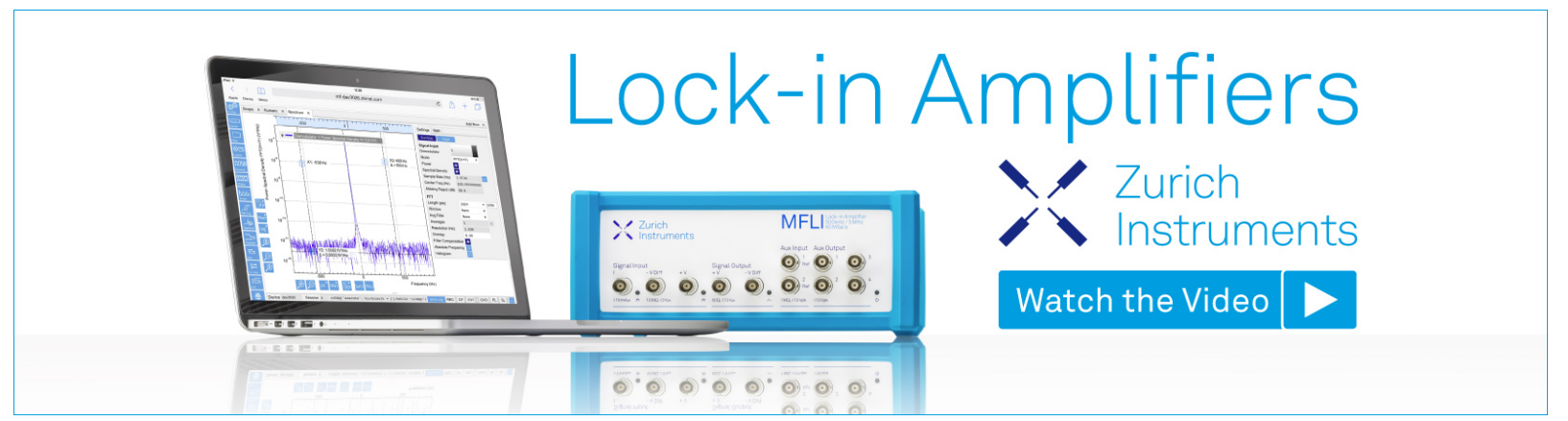




\title{
Amplified spontaneous emission and lasing in conducting polymers and fluorescent dyes in opals as photonic crystals
}

\author{
K. Yoshino, ${ }^{\text {a) }}$ S. Tatsuhara, Y. Kawagishi, and M. Ozaki \\ Department of Electronic Engineering, Graduate School of Engineering, Osaka University, Yamada-Oka, \\ Suita, Osaka 565-0871, Japan
}

\begin{abstract}
A. A. Zakhidov
Department of Thermophysics, Uzbek Academy of Sciences, Katartal 28, Chilernzar C, Tashkent 700135, Uzbekistan
\end{abstract}

Z. V. Vardeny

Physics Department, University of Utah, Salt Lake City, Utah 84112

(Received 20 November 1998; accepted for publication 8 March 1999)

\begin{abstract}
Spectral narrowing of photoluminescence (PL) and evolution of sharp emission lines upon optical excitation have been observed in opals made of $\mathrm{SiO}_{2}$ spheres infiltrated with conducting polymers such as OOPPV and MDDOPPV and also fluorescent dyes such as rhodamine 6G, NK-3483, and coumarin 120. Their emission properties are dependent on the sort of the opal and the solvents used for infiltration. With increasing optical excitation intensity, spectral narrowing and evolution of sharp lines have been observed in the green opal infiltrated with OOPPV, MDDOPPV, or rhodamine 6G but not in the infiltrated red and purple opals. With a solvent having a refractive index similar to that of $\mathrm{SiO}_{2}$, the evolution of the sharp emission lines is greatly suppressed and the lines exhibit a blueshift with decreasing refractive index. In NK-3483 and coumarin 120 which show red and purple PL, respectively, evolution of sharp lines is observed when they were infiltrated in red and purple opals, respectively, but not in other opals. These results are discussed in terms of amplified spontaneous emission and multimode lasing due to optical feedback in the opal matrix with a periodic structure. (C) 1999 American Institute of Physics. [S0003-6951(99)03218-0]
\end{abstract}

Recently, photonic crystals with three-dimensional regular structures of the periodicity of the order of the optical wavelength have attracted much attention because of theoretical prediction of concepts such as a photonic band gap ${ }^{1,2}$ and proposals of various applications of photonic crystals., However, compared with extensive theoretical studies, the experimental studies are rather limited. Synthetic opal with well-ordered arrays of $\mathrm{SiO}_{2}$ spheres in three dimensions is an interesting material from the point of view of a photonic crystal. We have reported that various organic materials such as dyes, conducting polymers, and liquid crystals can be infiltrated into the regular structure of interconnected nanoscale void space in these synthetic opals as photonic crystals and interesting optical properties have been demonstrated. ${ }^{5}$

On the other hand, light emitting diodes based on conducting polymers and fluorescent dyes have extensively been studied. ${ }^{6-9}$ We reported spectral narrowing and lasing in conducting polymers under intense optical excitation ${ }^{10}$ and in microcavities. ${ }^{11,12}$

In this letter, we report experimental studies on spectral narrowing of photoluminescence (PL) in conducting polymers and fluorescent dyes infiltrated in synthetic opals as photonic crystals.

Porous opals fabricated by sedimentation of the suspension of monodispersed $\mathrm{SiO}_{2}$ spheres 160,210 , and $250 \mathrm{~nm}$ in diameter and subsequent annealing at $100-120^{\circ} \mathrm{C}$ and sintering at $600-700{ }^{\circ} \mathrm{C}$ were used in this study. These opals

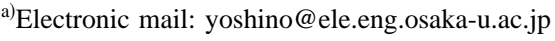

were dubbed purple opal, green opal, and red opal, respectively, because of their opalescent colors. In this experiment, an opal in the shape of a plate with a typical size of $10 \times 5 \times 1$ $\mathrm{mm}$ was used. These opals contain interconnecting tetrahedral and octahedral voids whose diameters depend on the $\mathrm{SiO}_{2}$ sphere diameters. The percolated porous structure permits the infiltration of conducting polymers and dye molecules as was already reported in our previous paper. ${ }^{5}$

Infiltration was carried out by dipping the opals into a conducting polymer solution such as poly(2,5-dioctyloxy-pphenylenevinylene) (OOPPV) and poly(2-methoxy-5dodecyloxy-p-phenylenevinylene) (MDDOPPV) and dyes such as rhodamine 6G, NK-3483, and coumarin 120 of appropriate concentrations. To study the effect of the refractive index, various solvents such as methanol, nitrobenzene, and their mixtures in the case of rhodamine $6 \mathrm{G}$, and toluene, tetrahydrofuran (THF), and their mixtures in the case of MDDOPPV were used.

The reflection spectrum was evaluated using a PMA11 charge coupled device (CCD) detector (Hamamatsu Photonics). Second harmonic generation (SHG) light $(532 \mathrm{~nm}$ in wavelength) of a Nd:YAG laser (1064 $\mathrm{nm}$ in wavelength) of $10 \mathrm{~ns}$ pulse width and a repetition rate of $2 \mathrm{~Hz}$ was used as an exciting light source for observations of spectral narrowing and lasing. The laser light irradiated the sample perpendicular to its flat surface. The emission spectra from the infiltrated opals were measured from the back side of the opal plate utilizing a MultiSpec257 spectrograph with a CCD detector (Oriel) having spectral resolution of $0.3 \mathrm{~nm}$.

Figure 1 shows reflection spectra of red, green, and 


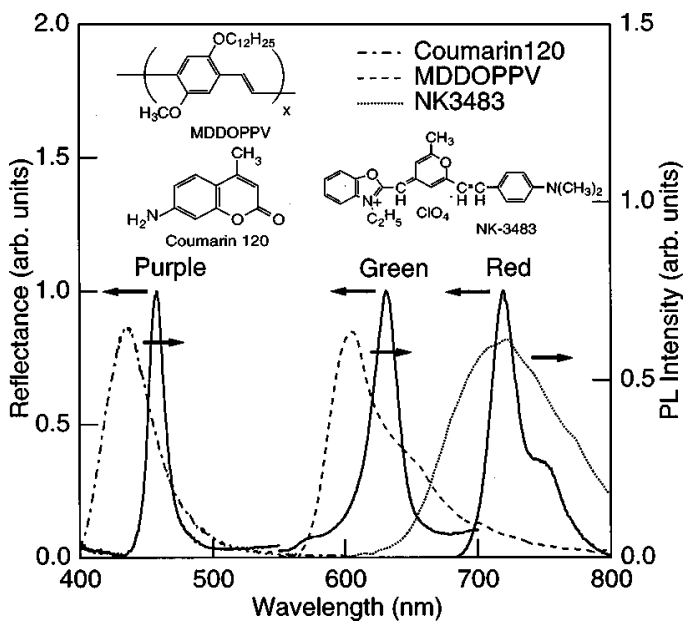

FIG. 1. Reflection spectra of synthetic opals: purple opal in methanol, green opal in toluene, and red opal in nitrobenzene, and PL spectra of MDDOPPV in a green opal, NK-3483 in a red opal, and coumarin 120 in a purple opal.

purple opals in solvents and PL spectra of solution of MDDOPPV, NK-3483, and coumarin 120 in which the molecular structures are also shown. As is evident, a clear refraction peak of green opal overlaps the PL from MDDOPPV. On the other hand, refraction peaks of the red and purple opals overlap the PL of NK-3483 and coumarin 120 , respectively.

Figure 2(a) shows the emission spectra of MDDOPPV in green opal infiltrated utilizing toluene as the solvent (5 $\left.\times 10^{-2} \mathrm{~mol} / \mathrm{l}\right)$. At low excitation level a wide spectral width of PL was observed peaking at around $598 \mathrm{~nm}$. As can be seen, with increasing excitation intensity, remarkable spectral narrowing is observed at $596 \mathrm{~nm}$ and simultaneous sharp emission lines with narrow spacing evolved at around 600$610 \mathrm{~nm}$. At higher excitation intensity, the enhancement of the spectrally narrowed peak was more remarkable than that of the sharp lines. Figure 3 shows the excitation intensity dependencies of the intensities of the spectrally narrowed peak and a newly evolved sharp emission line.

In the case of MDDOPPV in solution (for example, in

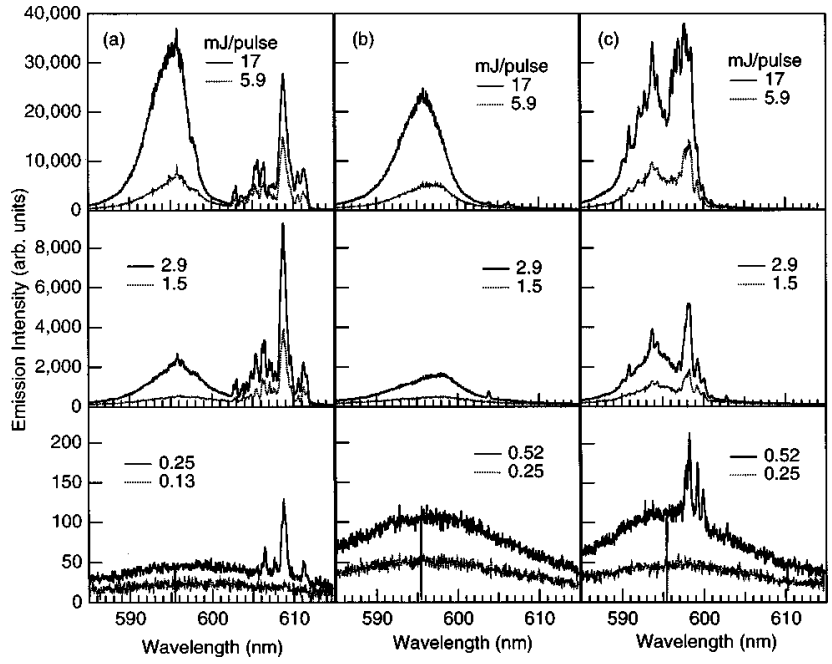

FIG. 2. Excitation intensity dependencies of emission spectra of MDDOPPV infiltrated in the green opal with solvents having refractive indices of (a) 1.50, (b) 1.45, and (c) 1.40. The concentration of MDDOPPV in solvent is $5 \times 10^{-2} \mathrm{~mol} / \mathrm{l}$.

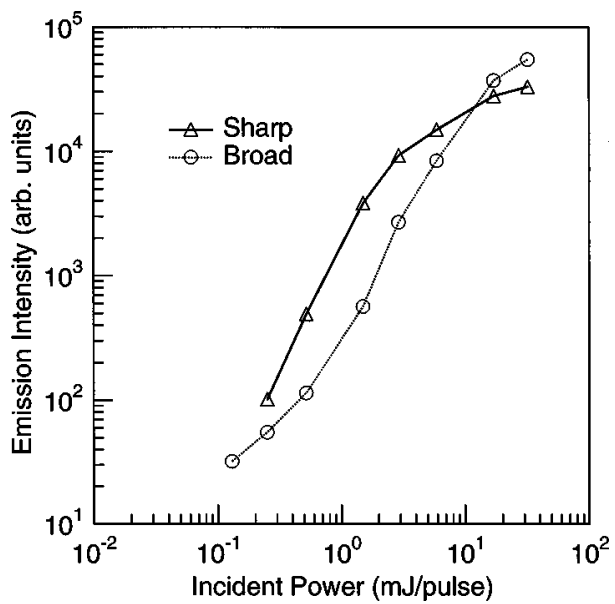

FIG. 3. Excitation intensity dependencies of intensities of the spectrally narrowed peak and a newly evolved sharp emission line of MDDOPPV infiltrated in a green opal with $5 \times 10^{-2} \mathrm{~mol} / \mathrm{l}$ in toluene.

toluene) but not in the opal matrix spectral narrowing of PL was observed at much higher excitation intensities than in the case in the opal matrix and the evolution of sharp lines was not observed.

It should also be noted that the MDDOPPV infiltrated with the same solvent in the red and purple opals did not remarkably exhibit the evolution of sharp emission lines. As evident in Fig. 1 the reflection peak of the green opal in toluene overlaps the PL of MDDOPPV in toluene. However the reflection peaks of the red and purple opals in toluene do not overlap the PL of MDDOPPV in the same solvent. This indicates that for, the appearance of sharp emission lines, the PL spectrum needs to overlap the spectral range of the reflection of the opal matrix. That is, the reflection due to the periodic structure of the opal plays an important role.

Similar spectral narrowing of PL and evolution of sharp lines of emission at high optical excitation were also observed in the green opal infiltrated with OOPPV and rhodamine $6 \mathrm{G}$ in which the PL peaks overlap the spectral range of the effective reflection in the green opal. In these cases the reflection peaks of the green opal in toluene and methanol overlap the PL of OOPPV in toluene and rhodamine $6 \mathrm{G}$ in methanol, respectively.

On the other hand, in the cases of NK-3483 and coumarin 120 which exhibit red and purple PL, respectively, the evolution of sharp emission lines was observed when they were infiltrated in the red and purple opals, respectively, as shown in Figs. 4(a) and 4(b). However, such sharp emission was not observed for NK-3483 in the green and purple opals or for coumarin 120 in the red and green opals. In these cases, the diffraction peaks of the red and purple opals overlap the PL peaks of NK-3483 and coumarin 120, respectively, which also supports the important role of optical feedback due to the matching of the optical periodicity of the opal matrix with the emission wavelength.

It should be mentioned that those spectral changes with excitation intensity were found to be dependent on the refractive index of the solvents used for infiltrations. As evident in Figs. 2(a)-2(c), when the refractive index of the solvent used for infiltration was similar to that of opal, $\mathrm{SiO}_{2}$, spectral narrowing was observed at a higher excitation level 

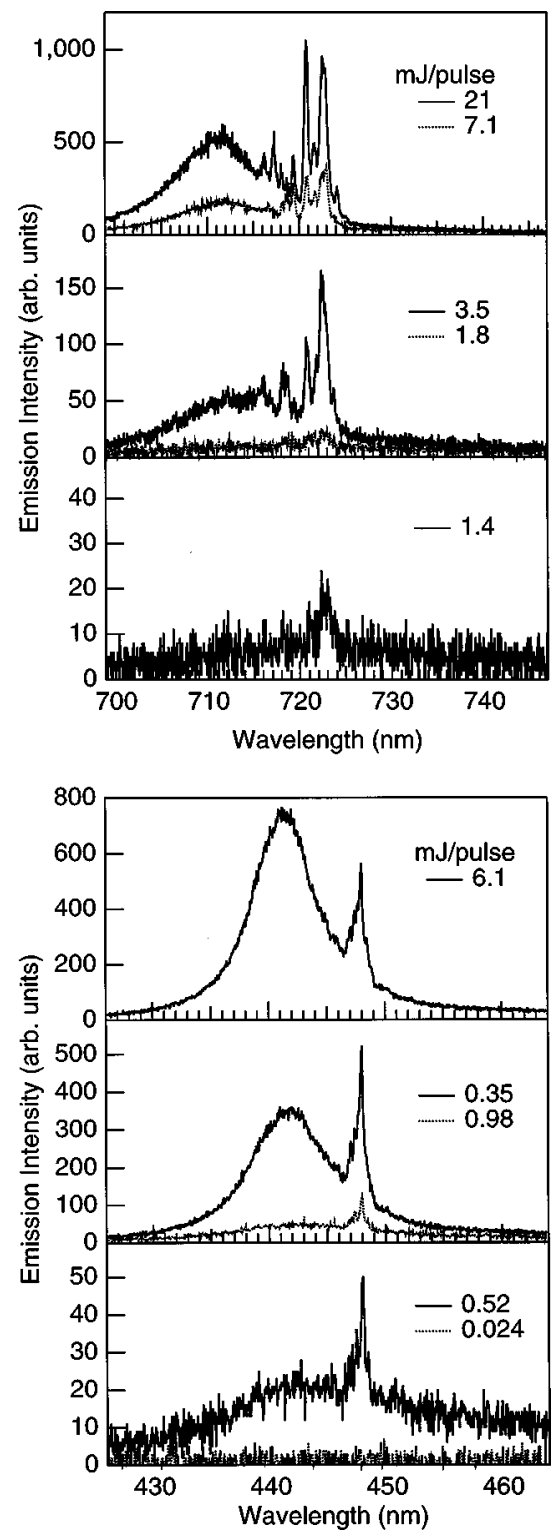

FIG. 4. (a) Emission spectra of the red opal infiltrated with NK-3483 of 5 $\times 10^{-3} \mathrm{~mol} / \mathrm{l}$ in nitrobenzene at various excitation intensities. (b) Emission spectra of the purple opal infiltrated with coumarin 120 of $1 \times 10^{-2} \mathrm{~mol} / \mathrm{l}$ in methanol at various excitation intensities.

but the evolution of sharp lines was greatly suppressed. It should also be noted that the wavelength of the sharp emission lines blueshifted with decreasing refractive index of the solvent, as evident in Figs. 2(a)-2(c). That is, in the solvent having a refractive index of 1.50 the sharp lines exhibited a blueshift of around $10 \mathrm{~nm}$ compared with the solvent of the refractive index of 1.40 . On the other hand, by changing the refractive index of the solvent from 1.40 to 1.50 the spectrally narrowed PL peaks exhibited only a small blueshift, less than 1-2 nm, which is comparable to the shift of the ordinary PL peak with the solvent. These facts indicate that the origin of the evolution of new sharp lines is different from that of spectral narrowing.
The remarkable spectral narrowing of PL observed may be explained in terms of amplified spontaneous emission (ASE). On the other hand, the sharp emission lines which were observed in the case of spectral overlapping of the PL and reflection of the opal may be explained by multimode laser emission on account of the optical feedback in the periodic structure of the opal matrix. The blueshift of the sharp emission lines' wavelength with the solvent's refractive index should reflect the change of the effective optical path length in the opal.

The possibility of random lasing may also exist. However, even in such a case, multiple coherent Bragg scattering of light in the periodic structures of materials seems to decrease the threshold compared with simple random scattering.

The present experimental study can be summarized as follows. Spectral narrowing of PL and the evolution of sharp emission lines with increasing optical excitation intensity were observed in the green opals infiltrated with conducting polymers such as MDDOPPV and OOPPV and also with fluorescent dyes such as rhodamine $6 \mathrm{G}$ in which spectral overlapping of the PL and the reflection of the green opals exist. On the other hand, in case of NK-3483 and coumarin 120 which exhibit red and blue PL, sharp emission lines evolved only when they were infiltrated in the red and purple opals, respectively. In the case of refractive index matching between $\mathrm{SiO}_{2}$ and the solvent, the evolution of sharp emission lines was greatly suppressed. The sharp emission lines shifted to shorter wavelengths with decreasing refractive index. On the other hand, the spectrally narrowed peaks did not exhibit such a large blueshift.

The observed spectral narrowing and the evolution of sharp emission lines were interpreted in terms of ASE and multimode lasing due to optical feedback in the opal matrix of the periodic structure, respectively.

This work was supported by a NEDO International Joint Research grant.

${ }^{1}$ S. John, Phys. Rev. Lett. 58, 2486 (1987).

${ }^{2}$ E. Yablonovitch, Phys. Rev. Lett. 58, 2059 (1987).

${ }^{3}$ S. John and T. Quang, Phys. Rev. Lett. 74, 3419 (1995).

${ }^{4}$ S. John and T. Quang, Phys. Rev. Lett. 78, 1888 (1997).

${ }^{5}$ K. Yoshino, K. Tada, M. Ozaki, A. A. Zakhidov, and R. H. Baughman, Jpn. J. Appl. Phys., Part 2 36, L714 (1997).

${ }^{6}$ C. W. Tang and S. A. VanSlyke, Appl. Phys. Lett. 51, 913 (1987).

${ }^{7}$ C. Adachi, T. Tsutsui, and S. Saito, Appl. Phys. Lett. 57, 531 (1990).

${ }^{8}$ J. H. Burroughes, D. D. C. Bradley, A. R. Brown, R. N. Marks, K. Mackay, R. H. Friend, P. L. Burns, and A. B. Holmes, Nature (London) 347, 539 (1990).

${ }^{9}$ Y. Ohmori, M. Uchida, K. Muro, and K. Yoshino, Jpn. J. Appl. Phys., Part 2 30, L1938 (1991); , 30, L1941 (1991).

${ }^{10}$ S. V. Frolov, M. Ozaki, W. Gellerman, Z. V. Vardeny, and K. Yoshino, Jpn. J. Appl. Phys., Part 2 35, L1371 (1996).

${ }^{11}$ S. V. Frolov, M. Shkunov, Z. V. Vardeny, and K. Yoshino, Phys. Rev. B 56, R4363 (1997)

${ }^{12}$ S. V. Frolov, Z. V. Vardeny, and K. Yoshino, Appl. Phys. Lett. 72, 1802 (1998). 